\title{
Gastric ESD may be useful as accurate staging and decision of future therapeutic strategy
}

\section{(1) $\circledast$}

\author{
Authors \\ Ai Fujimoto, Osamu Goto, Toshihiro Nishizawa, Yasutoshi Ochiai, \\ Joichiro Horii, Tadateru Maehata, Teppei Akimoto, Satoshi \\ Kinoshita, Seiji Sagara, Motoki Sasaki, Toshio Uraoka, Naohisa \\ Yahagi \\ Institutions \\ Center for Research and Development of Minimally Invasive \\ Treatment, Cancer Center, Keio University, Tokyo, Japan
}

submitted 20.4.2016

accepted after revision 4.10 .2016

Bibliography

DOI http://dx.doi.org/10.1055/s-0042-119392 |

Endoscopy International Open 2017; 05: EE90-EE95

(c) Georg Thieme Verlag KG Stuttgart · New York

ISSN 2364-3722

Corresponding author

Ai Fujimoto MD, PhD, 35 Shinanomachi, Shinjyuku-ku, Tokyo, Japan 160-8582

Phone: +81-3-5363-3895

Fax: $+81-3-5363-3895$

ai-fujimoto@a8.keio.jp

\begin{abstract}
Background and study aims We sometimes perform gastric endoscopic submucosal dissection (ESD) for total pathologic diagnosis when preoperative diagnosis is difficult. In the present study we analyzed the treatment outcomes and adverse events of diagnostic ESD for early gastric cancer (EGC).

Patients and methods We conducted a retrospective analysis of 18 consecutive cases of EGC in 18 patients with a suspected outof-indication diagnosis who underwent diagnostic ESD, between June 2010 and November 2014. The following parameters were examined: the average length of the longer axis of the lesion; the procedure time; the rates of en bloc resection (ER), complete en bloc resection (CER), and curative resection (CR) as treatment outcomes; and the rates of perforation, delayed bleeding, aspiration pneumonia, disease-related death, and emergency surgery as adverse events.

Results The treatment outcomes were as follows: average length of the longer axis of the lesion, $27.4 \pm 10.0 \mathrm{~mm}$; procedure time, $87.0 \pm 43.1$ minutes; ER rate, $18 / 18$ (100.0\%); CER rate, $13 / 18$ (72.2\%); CR rate, $4 / 18$ (22.2\%). CR rate was achieved $37.5 \%$ for the lesions which preoperative diagnosis was more than $30 \mathrm{~mm}$ $(>30 \mathrm{~mm})$ in diameter differentiated type with mucosal layer/submucosal layer 1 invasion and ulceration positive. The adverse events (AEs) were perforation in 1 of 18 (5.5\%) patients and delayed bleeding in 1 of 18 (5.5\%). There were no other AEs.

Conclusions Diagnostic ESD may be acceptable for future therapeutic strategy when we unconfirmed the pre ESD diagnosis because of lower rate of adverse events and high rate of ER.
\end{abstract}

\section{Introduction}

Endoscopic submucosal dissection (ESD) for early gastric cancer (EGC) has been widely performed around the world [1,2]. The rate of curative resection (CR) for lesions that meet the guideline of Japanese Gastric Cancer Association (JGCA) indications is as high as $97 \%$, which provides strong assurance that these lesions can be easily cured by ESD $[3,4]$. Technical progress with ESD has enabled en bloc resection (ER) for large and submucosal (SM) invasive lesions that were impossible to resect in the past $[5,6]$. Gotoda et al suggested that EGC with no risk of lymph node metastasis is definable by using a large database, so the indications for ESD have been expanded in Japan [7]. However, accurate assessment of depth of invasion is difficult in pre ESD lesion.

SM1 2 lesions, in particular are difficult because their invasion into the SM layer is slight. Tumor size more than $30 \mathrm{~mm}$, remarkable redness, uneven surface and margin elevation are characteristics of deeper SM cancer [8]. Even when using endoscopic ultrasonography (EUS), for example, the accuracy of es- timating the depth of cancer invasion is only $65 \%$ to $86 \%$ [9]. The important problem is the discrepancy in depth before and after ESD, which frequently occurs. When the diagnosis is difficult, empirical ESD as a diagnostic treatment may be a promising option.

In this study we evaluated the propriety of gastric ESD for total pathologic diagnosis of lesions which were endoscopically suspected as out-of-indication, but not definitely diagnosed before ESD.

\section{Patients and methods}

\section{Patients}

Among 287 cases of ESD due to EGC at our hospital between June 2010 and November 2014, 18 cases in 18 patients were suspected out-of-indication but not apparently diagnosed outof-indication according to endoscopic findings before the ESD. A retrospective study was performed on these cases based on their medical records. Patients with postoperative and residual stomach were excluded. All 18 cases were performed by ex- 
perts experienced with ESD in more than 1000 cases (N. Y, T.U, O.G, J.H. and Y.O.). Written informed consent was obtained from each patient.

\section{Definition of depth of cancer invasion for cMucosal layer (M)/SM1 or deeper than CSM2(SM massive)}

For superficial polypoid tumors (type $0-1$ ): pedunculated type (type $0-I \mathrm{p}$ ) or steep elevation in the $\mathrm{cM} / \mathrm{SM} 1$, sessile type (type $0-1 \mathrm{~s}$ ) or a gentle elevation of the lesion circumference mucosa such as a submucosal tunor (SMT); outstanding elevation or depression; disappearance of gastric area in the cSMmassive. For superficial slightly elevated tumor (type 0 - II a): Lesion diameter $<20 \mathrm{~mm}$ and gastric area present in the cM/SM1, outstanding depression or elevation; a node of uneven size; elevation on the depression side; hardness of the lesion circumference mucosa; and enlargement and fusion of folds in the cSMmassive [10]. For superficial slightly depressed tumor $(0-I \mathrm{c})$ with ulceration-negative (UL-) findings: depression side $<10 \mathrm{~mm}$; depression side flat in form; appearance of regular minute granules on the depression side with undifferentiated adenocarcinoma in the $\mathrm{CM} / \mathrm{SM} 1$, lesion diameter $>40 \mathrm{~mm}$; deep depression side; elevation on the depression side; irregular granules on the depression side; no gastric area on the depression side; significant redness; an elevation of lesion circumference mucosa such as an SMT; plateau elevation in the cSMmassive. For 0 - II c with ulceration-positive (UL+): decline or interruption of the folds in the $\mathrm{CM} / \mathrm{SM} 1$; enlargement and fusion of folds; an elevation of lesion circumference mucosa such as an SMT; thickness of lesion circumference folds and mucosa; plateau elevation in the cSMmassive [11].

Nagahama $T$ et al., meanwhile, identified a "non-extension sign" as a characteristic endoscopic sign of cSM2 [12]. In our study we diagnosed the depth of gastric cancer invasion as deeper than CSM2 according to the endoscopic sign mentioned above.

\section{ESD procedure}

ESD procedure was performed using a dual knife or hook knife (KD-650, KD-620, respectively; Olympus, Tokyo, Japan ), or a combination of the above through a 2-channel scope equipped with multibending and water jet functions (GIF-2TQ260M; Olympus Optical). A soft transparent hood (D-201-13404; Olympus, Tokyo, Japan) was attached to the tip of the endoscope to obtain good endoscopic views of the submucosal layer. Marking dots were placed on the normal mucosa at approximately $5 \mathrm{~mm}$ from the tumor margin to provide safety margins. After submucosal injection of glycerol (10\% glycerol and 5\% fructose; Chugai Pharmaceutical, Tokyo, Japan) with a small amount of indigo carmine and $0.1 \%$ epinephrine, a mucosal incision was made outside the marking dots. Hyaluronic acid solution was added to the injection solution when mucosal elevation was insufficient due to ulceration of the lesion or massive fibrosis of the submucosal layer $[13,14]$. After mucosal incision, dissection of the submucosal layer was performed and en bloc resection was able to be achieved completely. The resected specimen was cut into 4-mm-thick slices after formalin fixation. The histological type, size, depth of invasion, lateral and vertical margins, and lymphatic-vascular invasion were evaluated in each slice according to the Japanese Classification of Gastric Carcinoma [3].

\section{Indications of ESD}

Indications for ESD were determined by presence or absence of a risk of nodal metastasis and according to the gastric cancer treatment guidelines of the JGCA. The indication criteria were defined as differentiated-type mucosal cancer without UL, less than or equal to $20 \mathrm{~mm}$ in diameter $(\leq 20 \mathrm{~mm})$. Expanding-indication criteria were defined as follows: differentiated-type mucosal cancer without UL, irrespective of tumor size; differentiated-type mucosal cancer with $\mathrm{UL}, \leq 30 \mathrm{~mm}$; differentiatedtype minute (within $500 \mu \mathrm{m}$ from the musclaris mucosae) submucosal invasive cancer, $\leq 30 \mathrm{~mm}$; and undifferentiated-type mucosal cancer without UL, $\leq 20 \mathrm{~mm}$. Ot-of-indication criteria were defined as EGCs that did not meet the indication criteria or the expanded-indication criteria. Indication for ESD was judged by more than 2 endoscopists using white light endoscopy, chromoendoscopy with $2 \%$ acetic acid and indigo carmine, and narrow band imaging (NBI) magnified endoscopy. EUS was additionally used when the depth of the cancer invasion could not be assessed precisely. Histopathologic type was determined by biopsy before ESD. We decided indication ESD or not by group conference including ESD experts.

\section{Judgment of pathologic result}

ER was defined as resection in a single piece as opposed to piecemeal resection (in multiple segments). Complete en bloc resection (CER) was defined when en bloc resection was achieved, with tumor-negative margins. Curative resection (CR) was defined when CER was achieved, with absence of lymphovascular invasion. Unclear horizontal margin (HM) was analyzed as negative HM. Following are the guideline indication criteria and the expanded indication criteria for curative resection and of gastric ESD [3]. Guideline indication criteria: En-bloc resection, $\mathrm{HM}$, vertical margin (VM) 0 , lymphatic vessel invasion (ly) 0 , blood vessel invasion (v) 0 and $\leq 20 \mathrm{~mm}$, UL-, differentiatedtype, pT1a, Expanded indication criteria: En-bloc resection, HM0, VM0, ly0, v0, and 1) (>20 mm), UL(-), differentiatedtype dominant, pT1a (In the mixed-type, the component of the undifferentiated-type is $[<20 \mathrm{~mm}]) ; 2) \leq 30 \mathrm{~mm}$, UL(+), differentiated-type dominant, pT1a,; 3) $\leq 20 \mathrm{~mm}$, UL(-), undifferentiated-type dominant, pT1a; 4) $\leq 30 \mathrm{~mm}$, differentiated-type dominant, pT1b (SM1: less than $500 \mu \mathrm{m}$ ) (In the mixed-type, no component of the undifferentiated-type is present at the tip of the SM invasion).

\section{Evaluation}

The primary endpoint is to assess the ratio of the cases in which diagnostic ESD is useful. Furthermore, ER, CER, CR and adverse events were evaluated as the secondary endpoint.

The subjects were evaluated for the following treatment outcomes and adverse events: 1) Treatment outcomes - Average of longer axis of lesions, Average ESD procedure times, the rates of ER, CER, and CR. We defined as 'procedure time' from the incision start of the lesion to the end; and 2) Adverse events 
- Table 1 Preoperative diagnosis. ${ }^{1}$

\begin{tabular}{|c|c|c|}
\hline \multicolumn{2}{|l|}{ Preoperative diagnosis } & $\mathrm{n}=\mathbf{1 8}$ \\
\hline \multicolumn{2}{|l|}{ Deeper than SM2 $(n=6)$} & 6 \\
\hline \multirow[t]{4}{*}{ M/SM1 invasion $(n=12)$} & $>30 \mathrm{~mm}+\mathrm{UL}(+)$ & 8 \\
\hline & Undifferentiated-type +>20 mm & 1 \\
\hline & Undifferentiated-type + UL(+) & 2 \\
\hline & $>20 \mathrm{~mm}+\mathrm{UL}(+)+$ Undifferentiated-type & 1 \\
\hline
\end{tabular}

- Rates of perforation, delayed bleeding aspiration pneumonia, emergency surgery, and disease-related death. Delayed bleeding was defined as bleeding that required transfusion or surgical intervention, or bleeding that caused the hemoglobin level to decrease by $2 \mathrm{~g} / \mathrm{dL}$. In addition, we also reported the outcomes of non-CR cases.

\section{Results}

\section{Preoperative diagnosis}

The average patient age was $66.5 \pm 14.5$ and the male/female ratio was $16 / 2$.

Preoperative diagnoses were as follows: invasion penetrating deeper than the SM2, 6 cases; M/SM1 invasion, differentiated-type, $>30 \mathrm{~mm}$ with UL(+), 8 cases; M/SM1 invasion, undif-

$\checkmark$ Table 2 Outcomes of ESD.

\begin{tabular}{|c|c|}
\hline Outcomes & $n=18$ \\
\hline Average of longer axis of lesions (mm) & $27.4 \pm 10.0$ \\
\hline Average ESD procedure times (minutes) & $87.0 \pm 43.1$ \\
\hline$E R^{1}$ & $18 / 18(100 \%)$ \\
\hline $\mathrm{CER}^{1}$ & $13 / 18(72.2 \%)$ \\
\hline $\mathrm{CR}^{1}$ & $4 / 18(22.2 \%)$ \\
\hline
\end{tabular}

ferentiated-type and $>20 \mathrm{~mm}, 1$ case; M/SM1 invasion, undifferentiated type, with UL (+), 2 cases; M/SM1 invasion, undifferentiated-type and $>20 \mathrm{~mm}$ with UL $(+)$; 1 case ( $>$ Table 1 ). Only 1 case underwent EUS before the ESD procedure.

\section{Outcomes of ESD procedures}

The average values obtained were as follows: length of the longer axis of the lesions, $27.4 \pm 10.0 \mathrm{~mm}$; ESD procedure time, $87.0 \pm 43.1$ minutes; ER, 18/18 (100\%); CER, 13/18 (72.2\%); and CR, 4/18 (22.2\%) ( Table 2).

\section{Pathologic studies confirmed that CER was not achieved in 5 lesions}

VM positive, 3 cases; VM unclear, 2 cases ( $\triangleright$ Table 3 ). Additional surgery was performed for 3 cases, and there were no lymph node metastasis cases.

\section{Pathologic studies confirmed CR in 4 lesions ( Fig. 1)}

Preoperative diagnosis for 3 of 4 CR cases (75.0\%) was lesion for $>30 \mathrm{~mm}$ differentiated-type with M/SM1 and UL(+)' ( CR rate of lesion for $>30 \mathrm{~mm}$ differentiated-type with M/SM1 and $\mathrm{UL}(+)$ ' was $3 / 8(37.5 \%)$ ( $>$ Table 5$)$.

\section{Adverse events}

The adverse events (AEs) from ESD were perforation in 1 of 18 (5.5\%) patients and delayed bleeding in 1 (5.5\%). There were no cases of severe aspiration pneumonia, emergency surgery, and disease-related death ( $\triangleright$ Table 6 ).

- Table 3 Pathologic studies confirmed that CER was not achieved. ${ }^{1}$

\begin{tabular}{|c|c|c|c|}
\hline & & Preoperative diagnosis & Postoperative diagnosis \\
\hline 1 & VM unclear & $20 \mathrm{~mm} \geq \mathrm{SM} 2 \mathrm{UL}(-)$ diff. & 21 mm SM1 UL(-) tub1 \\
\hline 2 & VM positive & 15 mm $\geq$ SM2 UL(-) diff. & $15 \mathrm{~mm} \geq \mathrm{SM} 2 \mathrm{UL}(-)$ tub2 > por \\
\hline 3 & VM positive & $15 \mathrm{~mm} \geq \mathrm{SM} 2 \mathrm{UL}(-)$ diff. & $15 \mathrm{~mm} \geq \mathrm{SM} 2 \mathrm{UL}(+)$ tub2 > $1>$ por \\
\hline 4 & VM positive & $30 \mathrm{~mm} \geq \mathrm{SM} 2 \mathrm{UL}(-)$ diff. & $30 \mathrm{~mm} \geq \mathrm{SM} 2 \mathrm{UL}(-)$ por $>$ tub2 > sig \\
\hline 5 & VM unclear & $45 \mathrm{~mm} \geq \mathrm{SM} 2 \mathrm{UL}(-)$ diff. & $48 \mathrm{~mm} \geq \mathrm{SM} 2 \mathrm{UL}(-)$ tub2 \\
\hline \multicolumn{4}{|c|}{$\begin{array}{l}\text { CER, complete en bloc resection; HM, horizontal margin; VM, vertical margin; SM, submucosal layer; } \geq \text { SM2, deeper than or equal to SM2; UL, ulceration; diff., dif- } \\
\text { ferentiated type; tub1, well differentiated tubular adenocarcinoma; tub2, moderately differentiated tubular adenocaricinoma; por, poorly differentiated adenocar } \\
\text { cinoma; sig, signet-ring cell carcinoma. } \\
14 \text { in } 5 \text { cases were deeper than or equal to SM2 invasion. }\end{array}$} \\
\hline
\end{tabular}



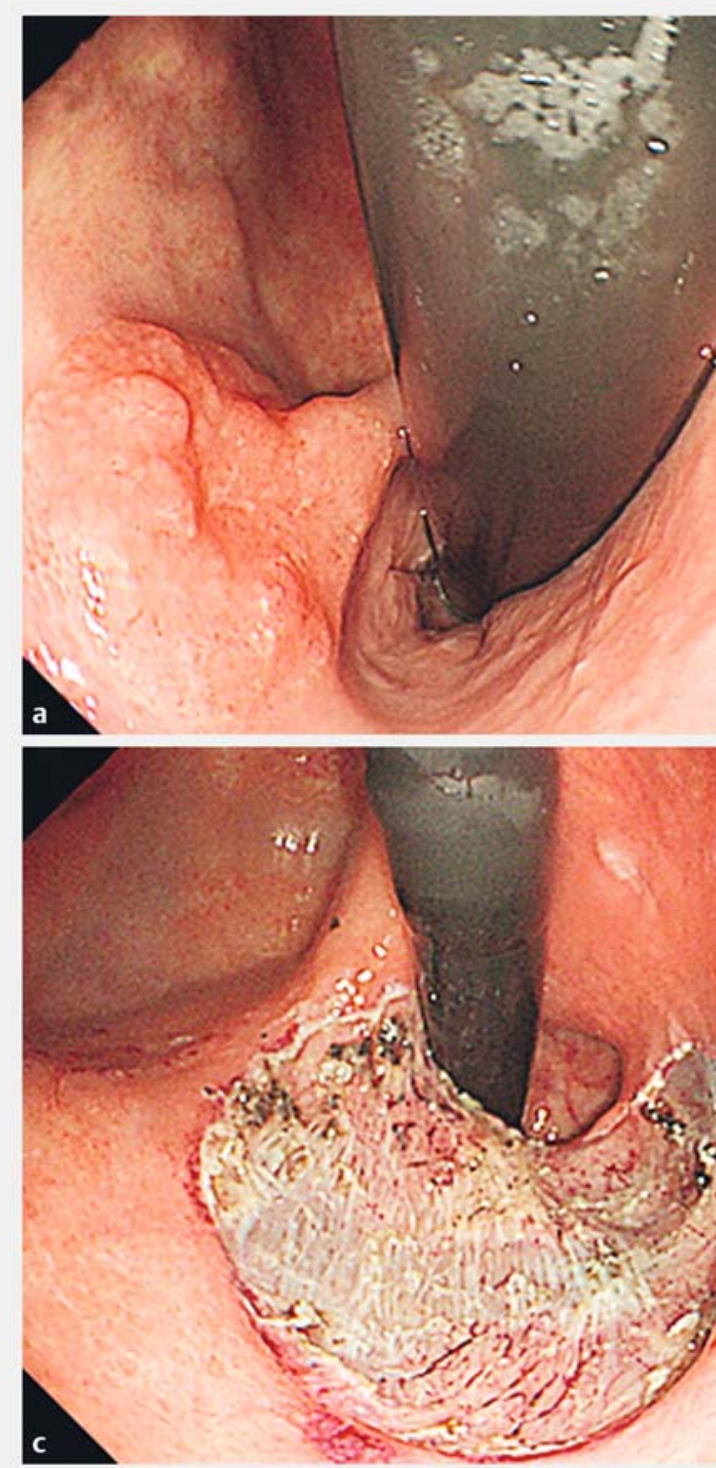
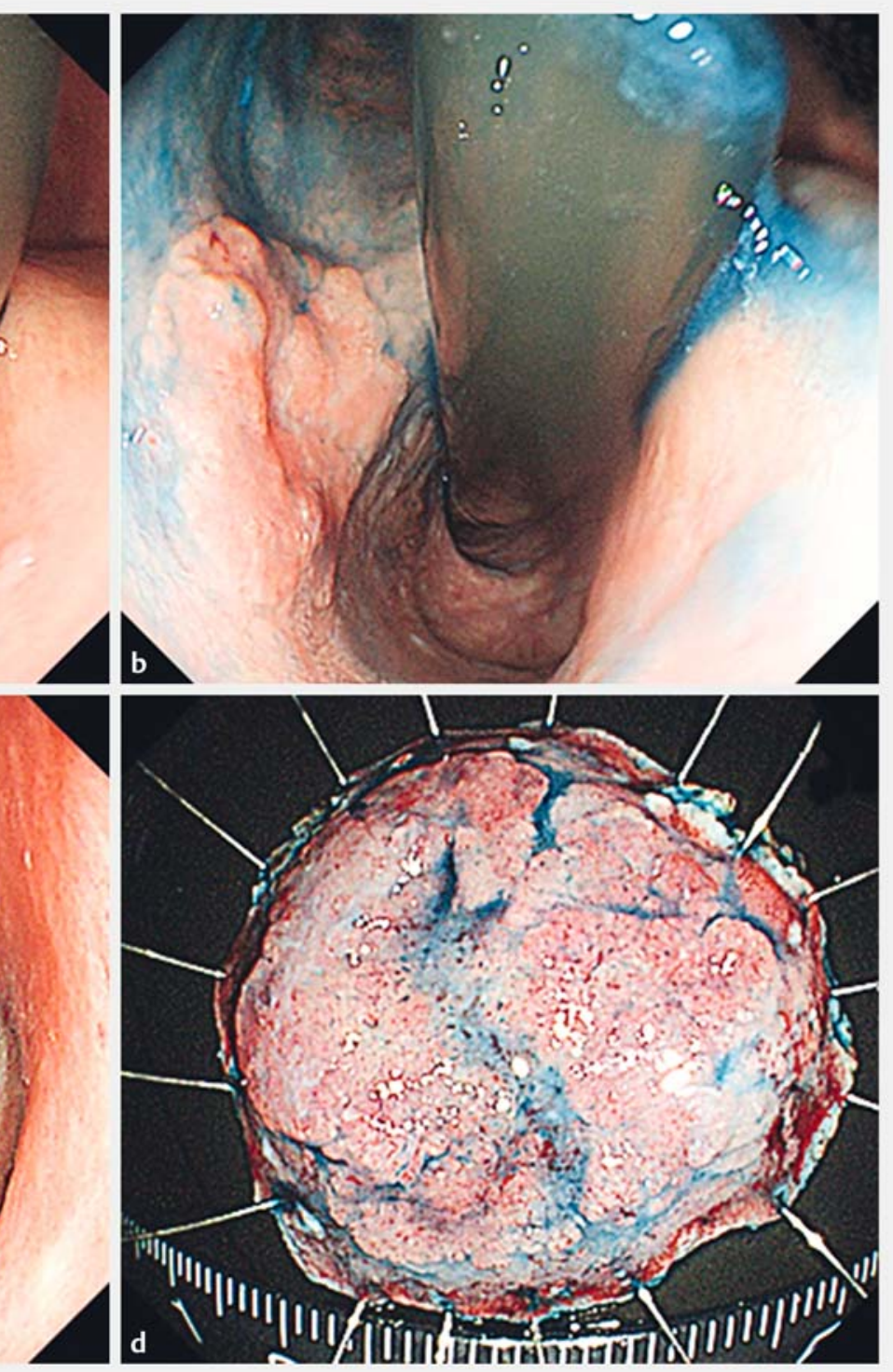

- Fig. 1 The estimated cancer invasion preoperatively was deeper than SM2, as elevations and depressions in the lesion were both clearly seen. The pathological finding, however, was SM1. a White light imaging. b Chromoendoscopy with $2 \%$ acetic acid and indigocarmine. $\mathbf{c}$ ESD ulcer. d ESD specimen.

- Table4 Pathologic studies confirmed to achieve CR.

\begin{tabular}{|c|c|c|}
\hline Case & Preoperative diagnosis ${ }^{1}$ & Postoperative diagnosis \\
\hline 1 & 35 mm M/SM1 UL(+) diff. & $30 \mathrm{~mm}$ adenoma \\
\hline 2 & 20 mm $\geq$ SM2 UL(-) diff. & 22 mm SM1 UL(-) tub1 > 2 ly0 v0 \\
\hline 3 & 35 mm M/SM1 UL(+) diff. & $12 \mathrm{~mm} \mathrm{M} \mathrm{UL(+)} \mathrm{tub1} \mathrm{ly0} \mathrm{v0}$ \\
\hline 4 & 31 mm M/SM1 UL(+) diff & $32 \mathrm{~mm}$ M UL(-) tub2 > tub1 ly0 v0 \\
\hline
\end{tabular}


- Table 5 CR rate.

\begin{tabular}{|c|c|c|}
\hline Preoperative diagnosis & & Curative resection rate \\
\hline Deeper than SM2 & 6 & $1 / 6(16.6 \%)$ \\
\hline$>30 \mathrm{~mm}$ M/SM1 differentiated-type with $\mathrm{UL}(+)^{1}$ & 8 & $3 / 8(37.5 \%)$ \\
\hline Undifferentiated-type with 1 positive among SM1, UL(+) or > $20 \mathrm{~mm}$ & 4 & $0 / 4(0 \%)$ \\
\hline
\end{tabular}

- Table6 6 Adverse events.

\begin{tabular}{|l|l|}
\hline Adverse event & \\
\hline Perforation & $1(5.5 \%)$ \\
\hline Delayed bleeding & $1(5.5 \%)$ \\
\hline Aspiration pneumonia & 0 \\
\hline Emergency surgery & 0 \\
\hline Disease-related death & 0 \\
\hline $\begin{array}{l}1 \text { There were no severe adverse events in the undiagnosed lesions that ap- } \\
\text { peared not to be indicated for ESD. }\end{array}$ \\
\hline
\end{tabular}

\section{Outcomes of 14 non-CR cases}

Additional surgery was performed in 10 of 14 (71.4\%) patients. Strict follow-up was performed every 6 months in 4 (28.6\%) patients. The average follow-up period for the patients who were strictly followed was $29.7 \pm 18.2$ months ( $6-57$ months). There were no cases of local recurrence, distance metastasis, or death related to gastric cancer. Among the 10 patients who underwent additional surgery, lymph node metastasis cases were not recognized. There were also no cases of residual cancer.

\section{Discussion}

To our knowledge, this is a first report of diagnostic gastric ESD that were performed for total pathologic diagnosis when preoperative diagnosis was difficult. ESD for EGC has proven to be a safe and effective treatment when it meets the guideline indications $[4,15]$. Progress in endoscopic devices and techniques now enables use of ESD not only for treatment, but also for overall pathologic diagnosis [16]. We sometimes perform ESD for total pathologic diagnosis when preoperative diagnosis is unconfirmed, as the precise depth of EGC invasion can be difficult to assess even with today's improved techniques for endoscopic diagnosis $[17,18]$.

Rapid local recurrence such as submucosal tumor was suspected in the future in 5 non-CER cases, as VM was either positive or unclear in the evaluations. Among these 5 patients in which VM was either positive or unclear, cancer invasion deeper than SM2 was confirmed in 4 patients and SM2 was suspected in all 5 patients preoperatively. When cancers are preoperatively estimated to invade deeper than SM2, the estimation is very likely to be correct. For cases of this type in the future, ESD will be supplanted by more appropriate methods such as endoscopic full-thickness resection corroborated by laparoscopic interventions such as non-exposed endoscopic wall-invasion surgery (NEWS) or full-layer resection for gastric cancer with nonexposure technique (CLEAN-NET) $[19,20]$.

On the other hand, CR was achieved in 4 (22.2\%) cases that were endoscopically suspected as out-of-indication before ESD. The endoscopic estimation of the depth of cancer invasion was deeper than the true depth in 1 of these 4 cases, and the 1 lesion was an adenoma. The accuracy in estimating the depth of cancer invasion appears to be limited. Rates of overstaging ranged from $11.1 \%$ to $24.1 \%$ even when we used EUS $[9,21]$. Furthermore, the differentiation between $M$ invasion and SM1 invasion by endoscopic finding is difficult to diagnose [9]. Diagnostic ESD for lesions $>30 \mathrm{~mm}$ of differentiated type with $\mathrm{M} /$ SM1 and UL(+) may be promising option. With regard to AEs, the rates of perforation and delayed bleeding were very low in the current study, matching the rates published in the ESD guideline [22]. Moreover, surgery is not always best choice for out-of-indication cases, considering the increased risk of surgery with comorbid diseases and the patient's quality of life after the surgery [23]. Although there is a risk of missing the opportunity for complete cure, "diagnostic ESD" and careful short-term follow-up may be possible strategy for well-selected patients.

Even lesions were suspected out-of-indication for ESD by preoperative diagnosis, $22.2 \%$ of them were taken with curative resection. In adition, with ER, $100 \%$ of the patients were cured and the incidence rate for accidental symptoms was also low. Consequently, when it is difficult to diagnose whether a lesion is susceptible to ESD, it is acceptable to perform ESD as diagnostic therapy.

\section{Competing interests}

None

\section{References}

[1] Neuhaus H. ESD around the world: Europe. Gastrointest Endosc Clin N Am 2014; 24: 295-311

[2] Kim MY, Cho JH, Jain P et al. ESD around the world: Asia. Gastrointest Endosc Clin N Am 2014; 24: $283-293$ 
[3] Japanese Gastric Cancer Association: Gastric cancer treatment guideline. 4th ed. Tokyo: Kanahara; 2014: $20-23$ (in Japanese)

[4] Toyonaga T, Man-i M, East JE et al. 1,635 Endoscopic submucosal dissection cases in the esophagus, stomach, and colorectum: complication rates and long-term outcomes. Surg Endosc 2013; 27: 1000 1008

[5] Gotoda T, Oda I, Tamakawa K et al. Prospective clinical trial of magnetic-anchor-guided endoscopic submucosal dissection for large early gastric cancer (with videos). Gastrointest Endosc 2009; 69: 10 15

[6] Lee WS, Cho JW, Kim YD et al. Technical issues and new devices of ESD of early gastric cancer. World J Gastroenterol 2011; 17: 3585-3590

[7] Gotoda T, Yanagisawa A, Sasako M et al. Incidence of lymph node metastasis from early gastric cancer: estimation with a large number of cases at two large centers. Gastric Cancer 2000; 3: 219-225

[8] Abe S, Oda I, Shimazu T et al. Depth-predicting score for differentiated early gastric cancer. Gastric Cancer 2011; 14: 35-40

[9] Yoshinaga S, Oda I, Nonaka S et al. Endoscopic ultrasound using ultrasound probes for the diagnosis of early esophageal and gastric cancers. World J Gastrointest Endosc 2012; 4: 218 - 226

[10] Sato S, Chonan A, Mishima T et al. Diagnosis of the Depth of Cancer Invasion for Early Gastric Cancer. Stomach and Intestine 2014; 49: $65-75$ (in Japanese)

[11] Maruyama Y, Shimamura T, Koda K et al. Diagnosis of the Depth of Early Gastric Cancer by Conventional and Dying Endoscopy from the View Point of the Size and Macroscopic Type. Stomach and Intestine 2014; 49: 35 - 46 (in Japanese)

[12] Nagahama T, Yao K, Imamura K et al. Diagnostic performance of conventional endoscopy in the identification of submucosal invasion by early gastric cancer: the "non-extension sign" as a simple diagnostic marker. Gastric Cancer Epub 2016: DOI: 10.1007/s10120-0160612-6 [Epub ahead of print]

[13] Yamamoto H, Kawata H, Sunada K et al. Successful en-bloc resection of large superficial tumors in the stomach and colon using sodium hyaluronate and small-caliber-tip transparent hood. Endoscopy 2003: 35: $690-694$

[14] Fujisjiro M, Yahagi N, Kashimura K et al. Comparison of various submucosal injection solutions for maintaining mucosal elevation during EMR. Endoscopy 2004; 36: 579- 583

[15] Yamaguchi N, Isomoto H, Fukuda E et al. Clinical outcomes of endoscopic submucosal dissection for early gastric cancer by indication criteria. Digestion 2009; 80: $173-181$

[16] Suzuki H, Oda I, Nonaka $S$ et al. Is endoscopic submucosal dissection an effective treatment for operable patients with clinical submucosal invasive early gastric cancer? Endoscopy 2013; 45: 93 - 97

[17] Sano T, Okuyama Y, Kobori O et al. Early gastric cancer. Endoscopic diagnosis of depth of invasion. Dig Dis Sci 1990; 35: 1340 - 1344

[18] Ono H, Yoshida M. Endoscopic diagnosis of the depth of cancer invasion for gastric cancer. Stomach and Intestine 2001; 36: 334 - 340 (In Japanese with English abstract)

[19] Inoue H, Ikeda H, Hosoya T et al. Endoscopic mucosal resection, endoscopic submucosal dissection, and beyond: full-layer resection for gastric cancer with nonexposure technique (CLEAN-NET). Surg Oncol Clin N Am 2012; 21: 129-140

[20] Goto O, Takeuchi H, Kawakubo $\mathrm{H}$ et al. Feasibility of non-exposed endoscopic wall-inversion surgery with sentinel node basin dissection as a new surgical method for early gastric cancer: a porcine survival study. Gastric Cancer 2015; 18: 440 - 445

[21] Yanai H, Matsumoto Y, Harada T et al. Endoscopic ultrasonography and endoscopy for staging depth of invasion in early gastric cancer: a pilot study. Gastrointest Endosc 1997; 46: 212 -216

[22] Yamaguchi N, Isomoto H, Fukuda E et al. Clinical outcomes of endoscopic submucosal dissection for early gastric cancer by indication criteria. Digestion 2009; 80: 173-181

[23] Kakushima N, Hagiwara T, Tanaka M et al. Endoscopic submucosal dissection for early gastric cancer in cases preoperatively contraindicated for endoscopic treatment. United European Gastroenterol ] 2013; $1: 453-460$ 